\title{
Formation of Polyol-Fatty Acid Esters by Lipases in Reverse Micellar Media
}

\author{
Douglas G. Hayes* and Erdogan Gulari ${ }^{\dagger}$ \\ Department of Chemical Engineering, University of Michigan, Ann Arbor, \\ Michigan 48109-2136
}

\section{Received July 19, 1991/Accepted January 7, 1992}

content to shift thermodynamic equilibrium in favor of esterification over hydrolysis, and be heterogeneous or biphasic in nature to accommodate all media components and provide lipases with an interface, which in most cases enhances the enzymes' performance. We have employed reverse micellar media composed of nanometer-sized dispersions of aqueous or polar material in a lipophilic organic continuum formed by the action of surfactants/cosurfactants to fulfill these criteria. In addition, compared to alternate types of reaction media, reverse micellar media provide excellent enzymesubstrate contact due to its dynamic nature, is easy to prepare, and has a large amount of interfacial area. The properties of reverse micelles and their application in enzymology and biotechnology have been thoroughly reviewed. ${ }^{15}$

In the first two articles of this series we have investigated the esterification of fatty acid and monohydroxyl alkyl alcohols for production of flavor esters ${ }^{8}$ and esterification with glycerol and a positional-specific lipase to produce monoglycerides, which are important food emulsifiers. ${ }^{10}$ Our goal here is to extend the work of the latter article to esterification with other polyols. Besides producing emulsifiers and texturizers, such reactions have importance in polymerization, e.g., as monomer units for crosslinking ${ }^{6}$ and in production of optically active polymers which can be employed in chiral separations. ${ }^{16}$ Fatty acid esters of sugars are of particular importance as low-calorie fat substitutes, biodegradable detergents, and components of paints and varnishes. ${ }^{2,18,20}$ The positional and regioselectivity of lipases is especially desirable for this reaction since the ester's physical properties are quite dependent on its molecular structure. Here, fatty acid esterification will be explored with the polyol substrates ethylene glycol $\left(\mathrm{HOCH}_{2} \mathrm{CH}_{2} \mathrm{OH}\right)$, sugars, and their derivatives, such as glucose and mannitol and 2-monoglycerides. The latter reaction is of particular interest in synthesizing "mixed" triglycerides, which are important components of synthetic oils that are difficult to produce selectively by conventional means. The latter reaction also represents a step of the lipase-catalyzed interesterification reaction between, e.g., palm oil and stearic acid, to produce a cocoa butter substitute through inexpensive means. It may also help explain why yields of triglycerides for the reaction between glycerol and lauric acid in reverse micelles are low, as obtained by Fletcher et al..$^{4,19}$ 


\section{MATERIALS AND METHODS}

\section{Materials}

The surfactant sodium bis(2-ethylhexyl) sulfosuccinate (AOT) was purchased from Aldrich (Milwaukee, WI) and used without further purification. Moreover, its high degree of purity (reported to be above $99 \%$ ) was verified by ultraviolet spectroscopy. In addition, calibrations from infrared spectroscopy indicate the AOT is nearly anhydrous, with water content being at water-surfactant molar ratios, or $w_{0}$ values, between 0.2 and 0.3 . Two lipase types were employed in these reactions and used without further purification: the first, from Rhizopus delemar, was purchased from Fluka (Ronkokoma, NY); the second, from Candida cylindracea, was purchased from Sigma (St. Louis, MO). All other reagents used were of high purity, including 2-monopalmitin (purchased from Sigma) and glycerol (from U.S. Biochemical of Cleveland, $\mathrm{OH}$ ); for fatty acids, glycerides, and polyols, their high degree of purity was verified by gas chromatography. Deionized water was employed throughout.

\section{Methods}

All reactions were carried out similarly to that described previously by us ${ }^{8,10}$ First, a solution of surfactant (AOT was employed exclusively) and substrates in isooctane was prepared. Typical concentration ranges for AOT and fatty acid substrate each were from 100 to $200 \mathrm{mM}$. A brief period of shaking was required for incorporation of ethylene glycol into reverse micelles. Next, a small aliquot of a lipase solution in $50 \mathrm{mM}$ phosphate buffer solution (PBS) at a $\mathrm{pH}$ of $6.89 \pm 0.05$ was injected into the media to initiate the reaction; gentle agitation for durations of a few seconds to $5 \mathrm{~min}$ was required for their incorporation into reverse micelles, as indicated by the cloudiness-clarity transition. (As a note concerning aqueous $\mathrm{pH}$ values, the addition of lipase from $R$. delemar slightly increased the $\mathrm{pH}$ of PBS by 0.1 unit at the concentrations employed while the addition of lipase from C. cylindracea had no such effect.) All reactions reported here were operated at room temperature: $23.5 \pm 0.5^{\circ} \mathrm{C}$. The procedure for operating sugar esterification reactions is similar to that just described, except the sugar substrate was added to the medium as a component of the aqueous lipase solution. At selected times, aliquots of medium were withdrawn for kinetic analysis, for which capillary gas chromatography (GC) with trimethylsilane (TMS) ether derivitization was employed. An internal standard was also added to the derivitization solution for quantification of GC peak areas. The procedure for analysis and the equipment and reagents employed have already been described in detail. ${ }^{8}$ The enzyme quantities reported are in terms of "units" of activity, v'here a unit is defined as lipase's ability to esterify $1 \mathrm{~m} M$ lauric acid with $n$-butanol per hour in reverse micellar media at these initial conditions: $w_{0}=9.23$, [lauric acid $]=[$ AOT $]=100 \mathrm{mM},[n$-butanol $]=$ $250 \mathrm{mM}$, temperature $23.5 \pm 1.5^{\circ} \mathrm{C}$, and aqueous $\mathrm{pH}$ of 6.9. Kinetic trends and results reported here are repeatable, with errors being within $5 \%$ for those cases checked.

Phase diagrams for water/AOT/isooctane media containing fatty acid and ethylene glycol were determined experimentally through titrations of water (PBS) and glycol into organic solvent containing AOT and fatty acid. The titration endpoints were noted by visual observation of the cloudiness-clarity transition. Phase boundaries for a given lauric acid/AOT/organic solvent ratio and temperature are plotted in terms of $w_{0}$ and $g_{0}$, the (ethylene) glycol/AOT ratio. Thus, the procedure and means of displaying results is similar to that contained in our previous article for glycerol-containing media. ${ }^{10}$

\section{RESULTS AND DISCUSSION}

\section{Ethylene Glycol-Lauric Acid Esterification}

The reaction investigated in this section is the esterification of ethylene glycol and lauric (dodecanoic) acid in isooctane-based reverse micellar media formed by the surfactant AOT. To better understand how the two substrates interact with the micellar media and to demark the quantities of glycol and fatty acid permitted by the media for micelle formation, phase diagrams were determined. As was performed for glycerol-containing reverse micellar media ${ }^{10}$ phase boundaries separating monophasic reverse micellar media from two-phase media were estimated for constant ratios of surfactant-fatty acidorganic solvent and temperatures. An example of experimentally determined phase boundaries is depicted in Figure 1. Here, the boundaries are plotted in terms of the water-AOT molar ratio $w_{0}$, and the (ethylene) gly-

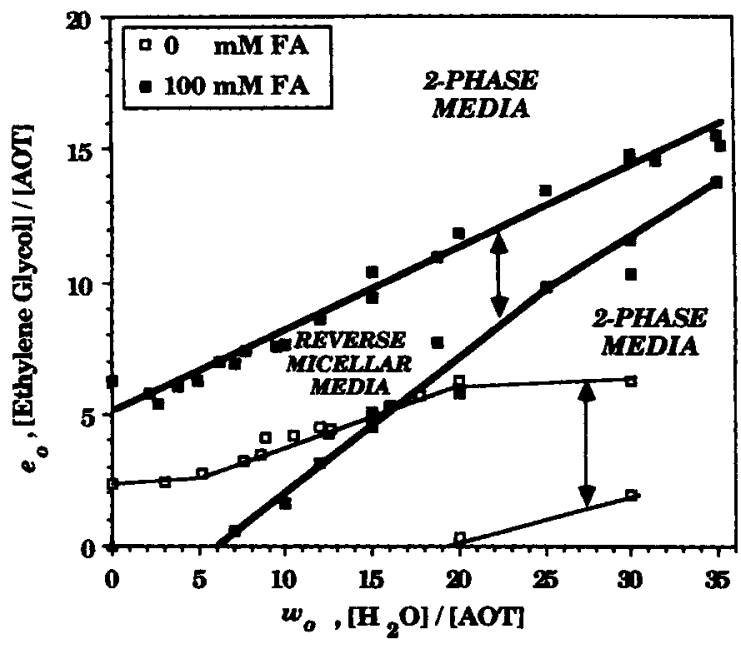

Figure 1. Effect of lauric acid concentration on the phase diagram for ethylene-glycol-containing reverse micellar media. The lines drawn are the lower and upper phase boundaries which separate the region where monophasic reverse micellar media exists from two-phase regions. Conditions: $T=24^{\circ} \mathrm{C}$ and $[\mathrm{AOT}]=100 \mathrm{mM}$. 
col-surfactant ratio $g_{0}$; this is in similar fashion to what was done previously. ${ }^{10}$ Note that glycol and water are the two species that solubilize inside the micellar droplets and hence $g_{0}$ and $w_{0}$ dictate micellar size., ${ }^{9,10}$ The figure illustrates that both an upper and lower phase boundary exists for ethylene-glycol-containing media, which is in contrast to the case of glycerol, where only an upper phase boundary was obtained..$^{10}$ The figure also demonstrates, when compared to data in our previous article, ${ }^{10}$ that much larger quantities of water and polyol can be solubilized when ethylene glycol replaces glycerol. Moreover, the solubilization of large quantities of glycol in hydrophobic organic solvent is an important finding. In addition, Figure 1 shows that solubilization of water and glycol as well as glycol and fatty acid are cooperative. We believe these relationships represent the cooperative action of agents that cause interfacial fluidity (e.g., glycol) and rigidity (e.g., fatty acid and ionic strength), where optimal solubilization exists when the two effects are balanced. Further experimental detail and discussion are contained elsewhere. ${ }^{9}$ For glycerol-containing media, the presence of glycerol reduced the maximum solubilization of water and fatty acid, ${ }^{10}$ which differs significantly from the trends illustrated for glycol.

The reaction between ethylene glycol $\left(\mathrm{HOCH}_{2}-\right.$ $\mathrm{CH}_{2} \mathrm{OH}$ ) and fatty acid (RCOOH) leads to synthesis of two products: a monoester, $\mathrm{RCOOCH} \mathrm{CH}_{2} \mathrm{OH}$ (abbreviated GME), and a diester, $\mathrm{RCOOCH}{ }_{2} \mathrm{CH}_{2} \mathrm{OOCR}$ (abbreviated GDE); a water molecule is also formed for each ester bond synthesized. Industrially, temperatures as high as $170-210^{\circ} \mathrm{C}$ are needed in the presence of alkaline catalysts to promote this reaction. ${ }^{2}$ But for the lipase-catalyzed reaction, conversions as high as $60 \%$ can be achieved in reverse micellar media at room temperature, as depicted in Figure 2. To achieve large conversions for the industrial reaction conditions, water must be continually removed. ${ }^{2}$ The figure demonstrates for our case that the reaction is near completion after 3-4 days at moderately low enzyme concentrations without water removal. Another important aspect of industrial application is the control of selectivity. Moreover, in many cases it is desirable to produce only the monoester since it is more valuable as an emulsifier than the diester. Industrially, to produce a high selectivity toward GME, a large excess of ethylene glycol is needed. ${ }^{2}$ Due to the large viscosity such a media would possess and the poor mass transfer that would be present, an expensive cosolvent would have to be included. ${ }^{2}$ But for the reaction in our media, Figure 2 demonstrates the product distribution can be composed almost solely of GME if the reaction is catalyzed by lipase from $R$. delemar and is stopped after 1-2 h without a huge excess of glycol. The overall percentage of conversion (of fatty acid, the limiting substrate) would be $20-30 \%$.

The major problem which must be solved for the employment of our reaction media industrially is the recovery of materials. The recovery of significant quantities of

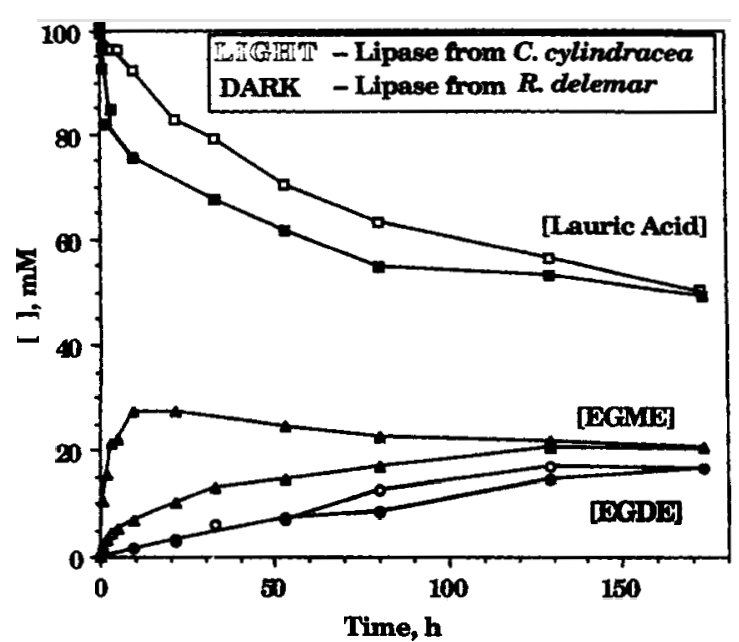

Figure 2. Substrate and product concentration changes with time for ethylene glycol-lauric acid esterification in reverse micelles at $24^{\circ} \mathrm{C}$ employing lipases from $C$. cylindracea (outlined symbols) at an overall concentration of $1.47 \mathrm{U} / \mathrm{mL}$ and $R$. delemar (darkened symbols) at $0.943 \mathrm{U} / \mathrm{mL}$. Initial conditions: $100 \mathrm{mM}$ AOT and lauric acid (in isooctane); $w_{0}=8.0$ and $g_{0}=3.0$; aqueous phase was $50 \mathrm{~m} M$ PBS at pH 6.89. ( $\square, \square)$ [lauric acid], $(\triangle, \Delta)$ [GME], and $(\bullet, 0)$ [GDE].

lipase activity from the media through extraction with a fresh aqueous phase at high ionic strength has been demonstrated. ${ }^{7}$ The separation of products from substrate can normally be achieved by simple techniques such as silica gel column chromatography or thin-layer chromatography. But the presence of the surfactant leads to poor separations by these techniques. ${ }^{7}$ We have investigated a simple extraction technique involving chloroform and methanol ${ }^{7}$ for separation of AOT and glycol from fatty acid and esters. Results indicated that acceptable separations of AOT and polyol from fatty acid and esters could not easily be achieved. ${ }^{7}$ A better, simpler procedure for AOT removal needs to be developed or an adequate substitute for AOT needs to be discovered.

Figure 2 illustrates that although lipase from $R$. delemar catalyzes formation of GME much faster than GDE, lipase from $C$. cylindracea catalyzes each ester at a similar rate and thus would have little value for selectively producing monoester. The fact that the final concentrations of substrates and products are nearly identical for each enzyme type indicates that the reaction stoppage is due not to enzyme deactivation but to the onset of thermodynamic equilibrium. Moreover, biocatalyst types should not affect the equilibrium position given the site of the reaction in the media does not change and that each biocatalyst possesses the capability to catalyze the specific reaction. The final percentage of conversion of limiting substrate matches the maximum range of values that was obtained for glycerol with a positional-specific lipase (from $R$. delemar), ${ }^{10}$ but there the product distribution was composed almost entirely of the monoester. The reason for this difference is yet unknown. In comparing the two enzymes, it can be seen that per-unit enzyme (as defined in the Materials and Methods sec- 
tion), lipase from $R$. delemar catalyzes ethylene glycolfatty acid esterification at a faster rate.

It is interesting that lipase from $R$. delemar can react with adjacent hydroxyl groups on an ethylene glycol molecule, whereas with glycerol, an ester bond at the middle, or 2, position of the polyol could not be formed. This illustrates that this lipase type catalyzes selectively due to steric hindrance effects at its active site. To explain, since ethylene glycol is only a two-carbon polyol, the monoester GME has sufficient flexibility and becomes extended and can easily penetrate into the active site. But at the middle carbon unit of glycerol, only one hydrogen group is contained and three bulky groups are attached, which leads to steric hindrance. Other evidence exists to support this postulate, including the fact that lipase from $R$. delemar cannot catalyze fatty acid esterification with 2-propanol while lipase from C. cylindracea, a nonpositional specific enzyme, can., ${ }^{7,8}$

Tables I-III describe the effect of $w_{0}, g_{0}$, and the initial fatty acid concentration, on the observed kinetics for the entire range of each variable allowed for the formation of monophasic reverse micellar media. Beginning with Table I, the results indicate the water content has only small effects on the reaction; moreover, initial rates are essentially invariant and percentage of conversion (of lauric acid) changes very little (except at $w_{0}=7$, where deactivation of lipase from $R$. delemar allows for formation of only trace amounts of GDE). This trend differs from the glycerol-fatty acid reaction, where larger water contents decreased the rate and extent of reaction ${ }^{10}$ and from lipase reactions in other organicsolvent-based media. ${ }^{5,21}$ Also, the table indicates that

Table I. Effect of $w_{0}$ on kinetics of lauric acid-ethylene glycol esterification in reverse micelles with lipase from $R$. delemar.

\begin{tabular}{|c|c|c|c|c|c|c|c|}
\hline \multirow{2}{*}{$\begin{array}{c}w_{0} \\
(\mathrm{~mol} / \mathrm{mol})\end{array}$} & \multicolumn{2}{|c|}{$[\mathrm{GME}]_{f}$} & \multicolumn{2}{|c|}{$[\mathrm{GDE}]_{f}$} & \multirow{2}{*}{$\begin{array}{c}\text { Percent } \\
\text { conversion of } \\
\text { lauric acid }\end{array}$} & \multirow{2}{*}{$\begin{array}{l}V_{\text {init }}^{\mathrm{GME}} /[\mathrm{E}]_{\mathrm{ow}} \\
\left(\frac{\mathrm{m} M}{\mathrm{~h}(\mathrm{U} / \mathrm{mL})}\right)\end{array}$} & \multirow{2}{*}{$\begin{array}{l}V_{\text {init }}^{\mathrm{GDE}} /[\mathrm{E}]_{\mathrm{ov}} \\
\left(\frac{\mathrm{m} M}{\mathrm{~h}(\mathrm{U} / \mathrm{mL})}\right)\end{array}$} \\
\hline & $\mathrm{m} M$ & $\%^{a}$ & $\mathrm{~m} M$ & $\%^{\mathrm{a}}$ & & & \\
\hline 7 & 24 & 100 & trace & 0 & 27 & 4.72 & 0 \\
\hline 10 & 27 & 46 & 16 & 52 & 63 & 6.07 & 0.175 \\
\hline 13 & 23 & 39 & 18 & 61 & 63 & 6.71 & 0.116 \\
\hline 16 & 21 & 38 & 17 & 62 & 56 & 6.26 & 0.165 \\
\hline 19 & 19 & 37 & 16 & 63 & 53 & 5.80 & 0.218 \\
\hline
\end{tabular}

Conditions as in Fig. 2 except $g_{0}=7$ and overall enzyme concentrations varied between $2.16 \mathrm{U} / \mathrm{mL}$ (for $w_{0}=7$ ) and $5.86 \mathrm{U} / \mathrm{mL}$ (for $w_{0}=19$ ).

${ }^{\mathrm{a}}$ Refers to percentage of product distribution.

Table II. Effect of $g_{0}$ on kinetics of lauric acid-ethylene glycol esterification in reverse micelles with lipase from $R$. delemar.

\begin{tabular}{|c|c|c|c|c|c|c|c|}
\hline \multirow{2}{*}{$\begin{array}{c}g_{0} \\
(\mathrm{~mol} / \mathrm{mol})\end{array}$} & \multicolumn{2}{|c|}{$[\mathrm{GME}]_{f}$} & \multicolumn{2}{|c|}{$[\mathrm{GDE}]_{f}$} & \multirow{2}{*}{$\begin{array}{c}\text { Percent } \\
\text { conversion of } \\
\text { lauric acid }\end{array}$} & \multirow{2}{*}{$\begin{array}{l}V_{\text {init }}^{\mathrm{GME}} /[\mathrm{E}]_{\mathrm{ov}} \\
\left(\frac{\mathrm{m} M}{\mathrm{~h}(\mathrm{U} / \mathrm{mL})}\right)\end{array}$} & \multirow{2}{*}{$\begin{array}{l}V_{\mathrm{init}}^{\mathrm{GDE}} /[\mathrm{E}]_{\mathrm{ov}} \\
\left(\frac{\mathrm{m} M}{\mathrm{~h}(\mathrm{U} / \mathrm{mL})}\right)\end{array}$} \\
\hline & $\mathrm{m} M$ & $\%^{a}$ & $\mathrm{~m} M$ & $\%^{a}$ & & & \\
\hline 1.5 & 11 & 44 & 7 & 56 & 20 & 4.78 & 0.077 \\
\hline 3.0 & 18 & 45 & 11 & 55 & 43 & 9.11 & 0.184 \\
\hline 4.5 & 21 & 43 & 14 & 57 & 56 & 11.8 & 0.238 \\
\hline 6.0 & 24 & 39 & 19 & 61 & 61 & 13.2 & 0.310 \\
\hline 7.5 & 31 & 56 & 12 & 34 & 60 & 13.0 & 0.186 \\
\hline
\end{tabular}

Conditions as in Fig. 2 except $w_{0}=10$ and overall enzyme concentration was $3.14 \mathrm{U} / \mathrm{mL}$.

${ }^{a}$ Refers to percentage of product distribution.

Table III. Effect of initial lauric acid concentration on kinetics of lauric acid-ethylene glycol esterification in reverse micelles with lipase from $R$. delemar.

\begin{tabular}{|c|c|c|c|c|c|c|c|}
\hline \multirow{2}{*}{$\begin{array}{c}{\left[_{\text {Laur Ac] }} \text { init }\right.} \\
(\mathrm{m} M)\end{array}$} & \multicolumn{2}{|c|}{$[\mathrm{GME}]_{f}$} & \multicolumn{2}{|c|}{$[\mathrm{GDE}]_{f}$} & \multirow{2}{*}{$\begin{array}{c}\text { Percent } \\
\text { conversion of } \\
\text { lauric acid }\end{array}$} & \multirow{2}{*}{$\begin{array}{l}V_{\text {init }}^{\mathrm{GME}} /[\mathrm{E}]_{\mathrm{ov}} \\
\left(\frac{\mathrm{m} M}{\mathrm{~h}(\mathrm{U} / \mathrm{mL})}\right)\end{array}$} & \multirow{2}{*}{$\begin{array}{l}V_{\text {init }}^{\mathrm{GDE}} /[\mathrm{E}]_{\mathrm{ov}} \\
\left(\frac{\mathrm{m} M}{\mathrm{~h}(\mathrm{U} / \mathrm{mL})}\right)\end{array}$} \\
\hline & $\mathrm{m} M$ & $\%^{a}$ & $\mathrm{~m} M$ & $\%^{\mathrm{a}}$ & & & \\
\hline 48 & 15 & 37 & 5.6 & 43 & 52 & 9.72 & 0.0829 \\
\hline 96 & 24 & 50 & 12 & 50 & 60 & 9.08 & 0.130 \\
\hline 143 & 28 & 37 & 24 & 63 & 58 & 7.74 & 0.106 \\
\hline 191 & 41 & 46 & 24 & 54 & 50 & 6.04 & 0.0509 \\
\hline
\end{tabular}

Conditions as in Fig. 2 except $w_{0}=10, g_{0}=5.0$, and overall enzyme concentration was $2.17 \mathrm{U} / \mathrm{mL}$.

${ }^{a}$ Refers to percentage of product distribution. 
the percentage of GDE in the product distribution increases slightly as $w_{0}$ is increased. In addition, the initial rates for ethylene glycol esterification contained in these three tables for lipase from $R$. delemar are in general at the same rate or at slightly faster rate than the ones for esterification with glycerol. ${ }^{10}$

Table II displays that unlike the effect of $w_{0}$, increases in $g_{0}$ lead to significant changes in initial rate-more specifically, to increases. Also, increases of $g_{0}$ improve the percentage of conversion but have little effect on the relative proportions of GME and GDE formed. An exception is for the last table entry, where it appears enzyme deactivation prematurely stops the reaction before significant quantities of diesters can be produced. The appearance of deactivation was supported by a series of experiments where enzyme concentration was the only reaction condition that was varied. For cases where the water content was low and the glycol and fatty content high, the reaction stopped shortly after initiation (even for high enzyme concentrations) and the final concentration of products was highest for experiments containing the largest enzyme concentrations. Conversely, at high water and glycol contents, no deactivation occurred. For example, at the initial conditions $g_{0}=15, w_{0}=35$, and [fatty acid] $=100 \mathrm{mM}$, the conversion was $60 \%$, with $20 \mathrm{~m} M$ each of GME and GDE produced. The trends discussed here are quite similar to those discovered for fatty acid-glycerol esterification in reverse micelles. ${ }^{10}$ The exact mechanisms for deactivation are unknown. We believe the cause for deactivation at high glycol/low water content is due to the high viscosity of the dispersed polar phase. At high fatty acid concentration, deactivation may be caused either by changes imposed on the micellar interface by fatty acids ${ }^{7}$ or by substrate inhibition.

The results for the effect of the initial fatty acid concentration are displayed in Table III. The table indicates that an increase in fatty acid concentration causes a slight decrease in the initial rate of product formation, which is opposite in trend for the increase in $g_{0}$. But unlike increases in $g_{0}$, increases in fatty acid slightly enhance the percentage of GDE in the product distribution. This compares to the effect of $w_{0}$ displayed in Table I.

The kinetic results obtained will now be compared to a simple model, which centers upon the formation of an acyl-enzyme intermediate. This intermediate is then subjected to nucleophilic attack by water, ethylene glycol, and GME. The derivation of the model is contained in the Appendix. Such a mechanism is supported experimentally ${ }^{13}$ and has been proven valid for esterification reactions in organic solvent catalyzed by suspended lipases. ${ }^{14}$ The model predicts a linear relationship between the quantity $[G]_{\text {init }} / V_{\text {init }}$ and $[G]_{\text {init }}$, for series of experiments where only the initial ethylene glycol content, $[G]_{\text {init }}$, is varied, such as the set contained in Table II. The results of the model fit are displayed in Figure 3 for

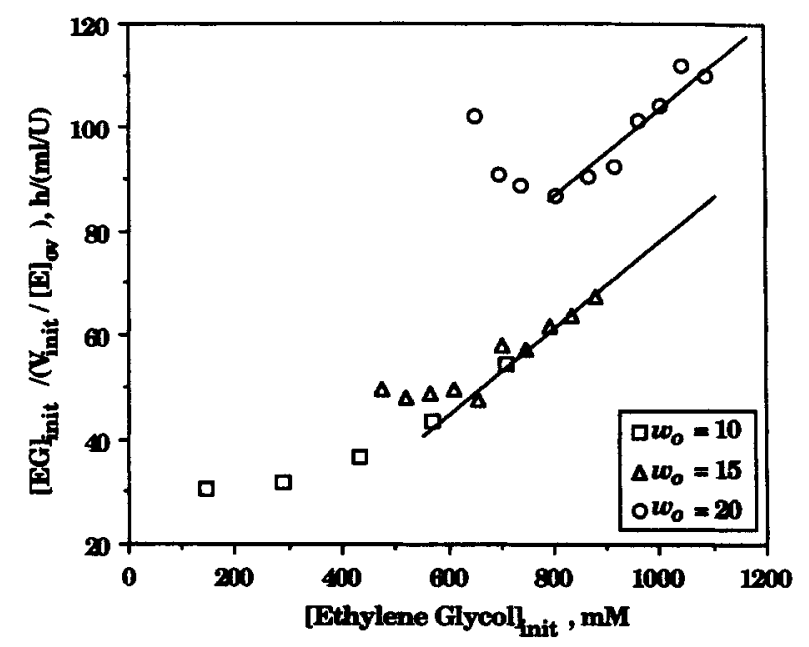

Figure 3. Fit of the kinetic model to data where the initial ethylene glycol concentration was varied. All reaction conditions are as in Fig. 2 except the enzyme type was exclusively lipase from $R$. delemar; water and ethylene glycol contents are as indicated in the figure.

three different initial water concentrations. As can be observed, the model predicts fairly well the kinetics at higher ethylene glycol concentrations but does not predict occurrences at low concentrations. This agrees with the other kinetic differences indicated by Table II for low initial ethylene glycol contents. Another interesting observation from the figure is that the slopes of the lines from the model for each of the three sets of experiments are nearly identical. This is consistent with the model, more specifically,

$$
\frac{V_{\max }[\mathrm{G}]}{V_{\text {init }}}=\left(1+\frac{k_{1}-[\mathrm{W}]}{k_{1}+[\mathrm{F}]}\right)+\left(\frac{k_{2-}}{k_{1}+[\mathrm{F}]}\right)[\mathrm{G}]
$$

[equals Equation (10) in the Appendix], which indicates the value of the slope should not be affected by the water concentration but the intercept value should increase. Indeed, the value of intercept does increase by the addition of water from $w_{0}=15$ to $w_{0}=20$. But between $w_{0}=10$ and $w_{0}=15$, no change in intercept values is apparent. Reasons for this discrepancy are yet unknown.

The model was also examined for the effect of initial water and fatty acid concentration. The results for the fit of the model to the data are poor. Moreover, the derivations contained in the Appendix indicate that the initial rate of monoester formation, $V_{\text {init }}^{\mathrm{GME}}$, should vary significantly with the initial water and fatty acid concentrations, but results such as those contained in Tables I and III indicate little variance exists. The failure of the model for the effect of water and fatty acid as well as the effect of glycol at low amounts is not surprising, as will now be demonstrated. To explain, the model employs not overall concentrations, but effective concentrations in the local environment of the catalyst. For lipase-catalyzed esterification reactions, the site of reaction is near the micellar interface. ${ }^{8}$ Thus, for the model to be completely accurate, partition coefficients for sub- 
strates between the two pseudophases and the interface need to be determined. For example, strong evidence exists from observation of physical phenomena with light scattering and phase diagrams for ethylene-glycolcontaining media that fatty acid partitions between the bulk organic phase and the micellar interface. ${ }^{9}$ In addition, it is obvious that the water content near the interface must be extremely low; thus, water concentrations should generally play only a minor role in the observed kinetics for esterification. The minor kinetic role of water content has been demonstrated by us also for esterification with monohydroxyl alcohols ${ }^{7,8}$ and with glycerol, except at conditions of large water concentration. ${ }^{10}$ To further explain the cause of this latter exception, at high water contents, smaller amounts of glycerol can be incorporated into the micelles due to the competition for solubilization between water and glycerol. ${ }^{10}$ This is why the cooperative solubilization of water and ethylene glycol is such an important result. Also, infrared spectroscopy results indicate that water displaces glycerol from the interfacial region. ${ }^{7,10}$ In similar fashion, the model deviations at low ethylene glycol concentrations demonstrated in Figure 3, which translate to slower rates of reaction than predicted, may be caused by water displacing interfacial glycol. Furthermore, since the model accurately predicts the effect of ethylene glycol concentration, the presence of glycol at the interface, i.e., the site of reaction, is confirmed.

In summary, the reaction between ethylene glycol and fatty acid proceeds readily in reverse micellar media, and unlike the case for glycerol, no significant dependence on the phase diagram exists. ${ }^{10}$ This is due primarily to ethylene-glycol-containing media being able to solubilize much larger quantities of water, which helps prevent enzyme deactivation, and polyol, which helps drive thermodynamic equilibrium favorably. In addition, the presence of ethylene glycol promotes interfacial fluidity, which improves enzyme stability. ${ }^{9}$

\section{Sugar-Fatty Acid Esterification}

The esterification reaction between fatty acid and sugars was investigated for each of the two lipase types listed above. For hexoses and their derivatives such as glucose and mannitol, no conversion was detected in reverse micelles for various sets of operating conditions. But results involving phase diagrams and light scattering suggest that the sugars interact with the micellar interface. Reasons for the reactions' failures are yet unknown; experimental details are contained elsewhere. ${ }^{7}$ Note that other researchers were able to obtain measurable conversion employing suspensions of lyophilized lipase in organic solvents, but the overall quantity of sugar esters produced was quite small. ${ }^{3}$

\section{Monoglyceride-Fatty Acid Esterification}

The specific reaction to be examined here is the esterification of 2-monopalmitin (2-hexadecanoylglycerol) and caprylic (octanoic) acid with lipase from $R$. delemar, a 1,3-positional specific biocatalyst. The objective with this reaction, as mentioned in the introduction, is to produce "mixed" glycerides, preferably, mixed triglycerides. The entire reaction is described in Figure 4. It shows, starting with 2-monopalmitin (2-MP), that esterification with caprylic acid (CA) can produce first 1-caprylic 2-palmitic diacylglycerol (abbreviated DG and referred to as "mixed" diglyceride) and then 1,3-caprylic 2palmitic triacylglycerol (abbreviated TG and referred to as "mixed" triglyceride); water is also produced at each of these two steps. However, our results indicate hydrolysis of 2-MP occurs also, since palmitic acid (PA) and glycerol are produced. Since lipase from $R$. delemar cannot cleave a bond at the middle hydroxyl of glycerol, it was assumed that 2-MP undergoes isomerization, and the resulting 1-MP is hydrolyzed by lipase. This scenario has been proven. To explain, a reaction media containing substrate and water, but not enzyme, was prepared and analyzed. The results indicate that, from 2-MP, 1-MP was formed in significant quantities, but no DG, TG, PA, or glycerol was formed. Thus, the isomerization step of Figure 4 occurs spontaneously, while all other steps, including hydrolysis of 1-MP, require the biocatalysis of lipase.

Figure 5 illustrates the change in concentration with time of substrates and products for esterification between 2-MP and CA. The figure indicates that DG is produced quite rapidly, and its concentration decreases after its maximum value is reached; during this period, TG is formed. Note this pattern is quite similar to the production of monoesters and diesters of ethylene glycol, as exhibited in Figure 2. Note also that the hydrolysis product PA (as well as glycerol) is formed. 1-MP is present throughout the reaction, but its concentration remains constant and is fairly small since it is rapidly hydrolyzed through the biocatalysis of lipase. In conclusion, Figure 5 indicates esterification occurs to a significant extent $(50 \%$ conversion of limiting substrate, 2-MP), but the major product is the diglyceride. Also, an undesirable hydrolytic side reaction occurs which depletes the concentration of 2-MP.

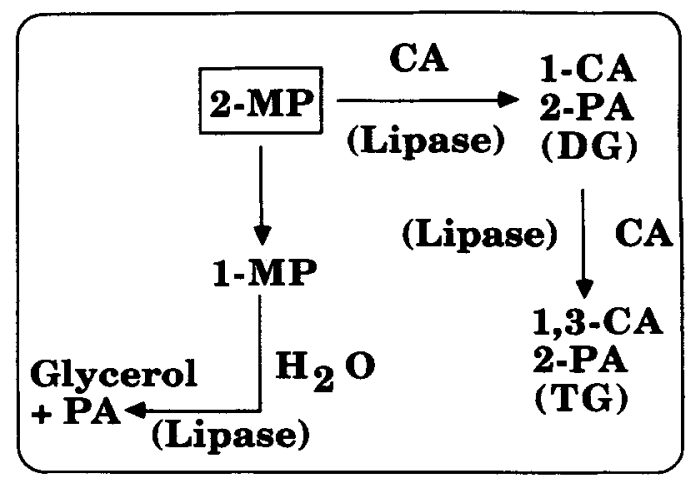

Figure 4. Reaction between 2-monopalmitin and caprylic acid by a 1,3-positional specific lipase. 


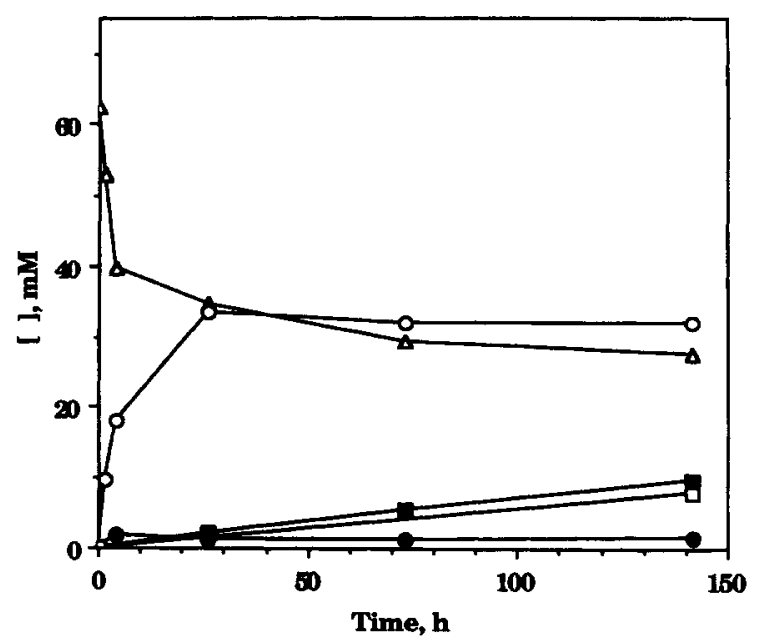

Figure 5. Changes in concentration of substrates and products for the esterification reaction between 2-monopalmitin and caprylic acid by lipase from $R$. delemar in reverse micellar media at $24^{\circ} \mathrm{C}$. Initial conditions: $[\mathrm{AOT}]=[\mathrm{CA}]=200 \mathrm{mM}, w_{0}=8.0$, and $[\mathrm{E}]_{\mathrm{ow}}=$ $2.8 \mathrm{U} / \mathrm{mL} .(\triangle)$ 2-MP, (O) DG, (ם) TG, ( $\square)$ PA, (๑) 1-MP. Aqueous phase was $50 \mathrm{~m} M$ PBS at $\mathrm{pH} 6.9$.

Table IV presents the effect of the initial 2-MP concentration on the extent of esterification. Of all the concentrations of media components, that of 2-MP was the most influential with respect to the extent of esterification. The table indicates that the amount formed and the percentage produced of DG and TG increase with the initial concentration of 2-MP, indicating its availability to the enzyme is what controls the kinetics. The table also demonstrates the extent of hydrolysis increases as well with larger 2-MP concentrations; but the relative ratio of products for esterification to hydrolysis remains constant or perhaps slightly increases. Again, the results of this table indicate the production of diglyceride is much greater than triglyceride. The probable cause of the small extent of TG synthesis is the small surface activity, i.e., amphiphilicity, possessed by diglyceride molecules in comparison to fatty acids and monoglyceride, as shown by light scattering. ${ }^{7}$ Thus, the local concentration of diglyceride in lipase's environment would be small. The rationale just discussed may help explain why synthesis of TGs in reverse micelles is difficult to achieve; for example, it would account for the small per-

Table IV. Effect of initial concentration of 2-MP on extent of esterification.

\begin{tabular}{|c|c|c|c|c|c|}
\hline $\begin{array}{l}{[2-\mathrm{MP}]_{\text {init }}} \\
\mathrm{mM}\end{array}$ & ${\frac{\mathrm{TG}^{\mathrm{a}}}{2-\mathrm{MP}}}^{\mathrm{a}}$ & $\frac{\mathrm{DG}}{2-\mathrm{MP}}^{\mathrm{a}}$ & $\frac{\mathrm{PA}}{2-\mathrm{MP}}^{\mathrm{a}}$ & $\frac{1-\mathrm{MP}^{\mathrm{a}}}{2-\mathrm{MP}}$ & $X^{\mathrm{b}}$ \\
\hline 8.6 & 0 & 28.7 & 11.4 & 5.8 & 1.7 \\
\hline 25.1 & 6.1 & 39.4 & 12.4 & 3.7 & 2.8 \\
\hline 41.5 & 6.9 & 40.2 & 12.5 & 2.5 & 3.1 \\
\hline 53.6 & 7.9 & 41.6 & 14.5 & 2.1 & 3.0 \\
\hline 69.4 & 10.0 & 43.0 & 13.9 & 2.2 & 3.3 \\
\hline
\end{tabular}

Conditions as in Fig. 5, except for the initial concentrations of 2-MP, which are listed in the figure.

a Times $100 \%$.

${ }^{b}$ The ratio of esterification to hydrolysis products. centage of conversion of TG formed from fatty acidglycerol esterification with a nonpositional specific lipase $^{4,19}$ and for the significant extent of hydrolysis reported for the transesterification between triacetin and tributyrin. ${ }^{1}$ Another research group has investigated the interesterification of stearic acid into palm oil in reverse micelles. ${ }^{11}$ They reported interesterification occurred to an extent as high as $30 \%$ but did not address whether hydrolysis of the triglycerides present occurred to a significant extent. ${ }^{11}$

Other experimentation was performed with the reaction between 2-MP and CA. For the effect of $w_{0}$ (i.e., the effect of AOT concentration at constant initial water concentration), the results indicate little variance with the extent of esterification and only a small effect on the rate of reaction; but the extent of hydrolysis increased significantly with an increase in $w_{0}$. This might be caused by larger water concentrations in the enzyme's environment at larger values of $w_{0}$. For the effect of the initial concentration of $\mathrm{CA}$, again, the extent and rate of esterification were not strongly affected. But the extent of hydrolysis decreased at larger amounts of CA. This trend may occur due to fatty acid reducing the amount of water bound to the interface, as discussed for glycerolcontaining media. ${ }^{7}$ Another explanation for the effects of fatty acid concentration and $w_{0}$ on the extent of hydrolysis is the effect imposed on the isomerization reaction by these changes. What causes isomerization for this type of reaction is yet unknown.

\section{CONCLUSIONS}

In this article, we have extended our research with lipasecatalyzed esterification in reverse micellar media to alternate polyol substrates-more specifically, ethylene glycol, monoglycerides, and sugars. Unfortunately, for the latter polyol, no esterification took place. With ethylene glycol as substrate, conversions were about 50-60\% at room temperature, which compares to results obtained for the reaction with glycerol in our previous report. ${ }^{10}$ But unlike the reaction with glycerol, here both monoand diesters were produced in significant amounts. Also, a product distribution of solely monoester can be achieved by employing lipase from $R$. delemar and stopping the reaction after a short time-about $20-30 \%$ conversion of substrate to monoester would be obtained. In addition, the capability of the media to solubilize large quantities of water and ethylene glycol reduces sensitivity to enzyme deactivation and reversal of thermodynamic equilibrium. Furthermore, a simple Michaelis-Menten kinetic model was developed which accurately describes the effect of ethylene glycol concentration on initial rate kinetics at most conditions but fails to describe the effect of water or fatty acid concentration due to the exclusion of partitioning effects.

For the reaction between 2-monopalmitin and caprylic acid catalyzed by the positional-specific lipase from 
$R$. delemar for "mixed" glyceride production, conversions toward esterification were as high as $50 \%$, but the product distribution was composed of mostly mixed diglyceride with only a small amount of mixed triglyceride present. Also, a significant amount of 2-monopalimitin spontaneously isomerized; the 1-monopalimitin formed was rapidly hydrolyzed by lipase from $R$. delemar. The major requirement for significant conversion is a large initial monoglyceride concentration.

Financial support for this project by the National Science Foundation is gratefully acknowledged.

\section{NOMENCLATURE}

Ac acyl group: $\mathrm{R}-\mathrm{COO}$, where $\mathrm{R}$ is an alkyl group (Appendix)

AOT surfactant Aerosol-OT

CA fatty acid caprylic (octanoic) acid

D fatty acid-glycol diester (Appendix)

DG "mixed" diglyceride (1-capryl 2-palmitoyl triacylglycerol)

E enzyme lipase (Appendix)

$E_{f} \quad$ "free enzyme," meaning enzyme unbound to acyl group (Appendix)

$E_{1} \quad$ total amount of enzymersee Eq. (6)

$(\mathrm{E} \cdot \mathrm{Ac})$ acyl-enzyme intermediate (Appendix)

$F \quad$ fatty acid (Appendix)

$G \quad$ ethylene glycol

GDE ethylene glycol-lauric acid diester

GME ethylene glycol-lauric acid monoester

$g_{0} \quad$ (ethylene) glycol- (or glycerol-) surfactant ratio $(\mathrm{mol} / \mathrm{mol})$

$k_{i} \quad$ rate constant for reation step $i$ (subscripts explained in Appendix)

M ethylene glycol-fatty acid monoester (Appendix)

MP monoglyceride monopalimitin

PA palmitic (hexadecanoic) acid

PBS phosphate buffer solution

TG "mixed" triglyceride (1,3-capryl 2-palmitoyl triacylglycerol)

$U \quad$ units of lipase activity

$V \quad$ velocity of reaction $(\mathrm{m} M$ product $/ \mathrm{h})$

$V_{\max } \quad$ maximal velocity which can be obtained, as defined in the Appendix

W water (Appendix)

$w_{0} \quad$ water-surfactant ratio $(\mathrm{mol} / \mathrm{mol})$

Subscripts and superscripts

init initial reaction conditions

M monoester of ethylene glycol

\section{APPENDIX}

This appendix contains the derivation of the kinetic model employed to describe the reaction between ethylene glycol and fatty acid. The derivation begins with a description of the reaction steps. The first step is the formation of an acyl-enzyme intermediate from fatty acid and lipase:

$$
\mathrm{AcOH}+\mathrm{E} \longleftrightarrow(\mathrm{E} \cdot \mathrm{Ac})+\mathrm{W}
$$

where Ac refers to the acyl group (thus, the fatty acid, denoted F, is $\mathrm{AcOH}$ ), $\mathrm{E}$ the enzyme (lipase), and $\mathrm{W}$ a water molecule. Note that the reaction is reversible. The two rate constants are $k_{1+}$ and $k_{1-}$ for the forward and reverse directions of Equation (2). The acyl-enzyme intermediate can undergo further reaction to produce esters:

$$
\begin{aligned}
& (E \cdot A c)+G \longleftrightarrow M+E+W \\
& (E \cdot A c)+M \longleftrightarrow D+E+W
\end{aligned}
$$

where $M$ and $D$ are mono- and diesters, respectively, and $G$ the glycol (ethylene glycol, e.g.). The constants involved, for forward and reverse directions, respectively, are $k_{2-}$ and $k_{2+}$ for Equation (3) and $k_{3-}$ and $k_{3+}$ for Equation (4). Thus, the model does not include glycol-enzyme intermediates and neglects the contribution of the acyl-enzyme-glycol complex; thus, it is assumed that the formation of ester products is relatively fast once nucleophilic attack occurs.

For each esterification product formed, a rate eqution can be derived; for example,

$$
\begin{aligned}
\frac{d[\mathrm{M}]}{d t}=k_{2-}[\mathrm{E} \cdot \mathrm{Ac}][\mathrm{G}]+k_{3+}[\mathrm{D}][\mathrm{E}]_{f}-k_{2+}[\mathrm{M}][\mathrm{E}]_{f} & \\
& -k_{3-}[\mathrm{E} \cdot \mathrm{Ac}][\mathrm{M}]
\end{aligned}
$$

The symbol $[E]_{f}$ refers to the concentration of "free" enzymes, which is defined by the following mass balance:

$$
[\mathrm{E}]_{f}=[\mathrm{E}]_{\imath}-[\mathrm{E} \cdot \mathrm{Ac}]
$$

Here, $[E]_{i}$ refers to the "total" enzyme concentration-the only quantity in Equation (6) which can be measured. The MichaelisMenten model is centered upon the pseudo-steady-state hypothesis for the enzyme-substrate intermediate:

$$
\begin{aligned}
& \frac{d[\mathrm{E} \cdot \mathrm{Ac}]}{d t} \approx 0=\left(k_{1+}[\mathrm{F}]+k_{2+}[\mathrm{M}]+k_{3+}[\mathrm{D}]\right)[\mathrm{E}]_{f} \\
&-\left(k_{1-}[\mathrm{W}]+k_{2-}[\mathrm{G}]+k_{3-}[\mathrm{M}]\right)[\mathrm{E} \cdot \mathrm{Ac}]
\end{aligned}
$$

Combining Equations (5)-(7) yields the following equation for the rate of monoglyceride production:

$$
\begin{aligned}
& \frac{d[\mathrm{M}]}{[\mathrm{E}]_{t} d t}=k_{3+}[\mathrm{D}]-k_{2+}[\mathrm{M}] \\
& +\frac{\left(k_{2-}[\mathrm{G}]+k_{2+}[\mathrm{M}]-k_{3+}[\mathrm{D}]-k_{3-}[\mathrm{M}]\right)\left(k_{1+}[\mathrm{F}]+k_{2+}[\mathrm{M}]+k_{3+}[\mathrm{D}]\right)}{k_{1+}[\mathrm{F}]+k_{2+}[\mathrm{M}]+k_{3+}[\mathrm{D}]+k_{1-}[\mathrm{W}]+k_{2-}[\mathrm{G}]+k_{3-}[\mathrm{M}]}
\end{aligned}
$$

Such equations as Equation (8) are quite complex and require simplification. The model will now focus specifically upon the initial rate of monoester formation. Initially, the amount of each monoand diester present is small; thus their concentrations will be set equal to zero. Applying these assumptions to Equation (8) leads to

$$
\left.\frac{d[\mathrm{M}]}{d t}\right|_{\text {init }} \equiv V_{\text {init }}^{\mathrm{M}}=\frac{[\mathrm{E}]_{2} k_{2-}[\mathrm{F}][\mathrm{G}]}{[\mathrm{F}]+\frac{k_{1-}}{k_{1+}}[\mathrm{W}]+\frac{k_{2-}}{k_{1+}}[G]}
$$

The quantity $[\mathrm{E}], k_{2-}$ will be given the symbol $V_{\max }$, which has significance in the Michaelis-Menten model as the maximum rate of reaction that can be obtained. Equation (9) can be easily manipulated to create the following expression:

$$
\frac{V_{\text {max }}[\mathrm{G}]}{V_{\text {init }}}=\left(1+\frac{k_{1-}[\mathrm{W}]}{k_{1+}[\mathrm{F}]}\right)+\left(\frac{k_{2-}}{k_{1+[}[\mathrm{F}]}\right)[\mathrm{G}]
$$

Thus, a plot of $[\mathrm{G}]_{\text {init }} / V_{\text {init }}$ versus $[\mathrm{G}]_{\text {init }}$ should yield a straight line if this model holds and if the initial water and fatty acid concentrations are held constant for all data. Likewise, Equation (9) can be manipulated into the following:

$$
\frac{V_{\max }[\mathrm{F}]}{V_{\text {init }}}=\left(\frac{k_{1-}[\mathrm{W}]}{k_{1+}[\mathrm{G}]}+\frac{k_{2-}}{k_{1+}}\right)+\frac{[\mathrm{F}]}{[\mathrm{G}]}
$$

Thus, for a series of kinetic experiments where only the initial fatty acid concentration is varied, a plot of $[\mathrm{F}]_{\text {init }} / V_{\text {init }}$ versus $[\mathrm{F}]_{\text {init }}$ should 
also yield a straight line. Furthermore, Equation (9) in the form

$$
\frac{V_{\text {max }}[\mathrm{F}][\mathrm{G}]}{V_{\text {init }}}=\left([\mathrm{F}]+\frac{k_{2-}[\mathrm{G}]}{k_{1+}}\right)+\left(\frac{k_{1-}}{k_{1+}}\right)[\mathrm{W}]
$$

indicates that for a series of experiments where only the water content is varied, a straight line should fit $1 / V_{\text {init }}$ versus $[\mathrm{W}]_{\text {init }}$.

The model was also investigated to determine whether such simplified expressions could be derived for the initial rate of diglyceride formation; no such expressions were determined.

\section{References}

1. Abraham, G., Murray, M. A., John, V.T. 1988. Interesterification selectivity in lipase catalyzed reactions of low molecular weight triglycerides. Biotechnol. Lett. 10: 555-558.

2. Benson, F. R. 1967. Polyol surfactants. pp. 247-299 In: M. J. Schick (ed.), Nonionic surfactants: surfactant science series, vol. 1. Marcel Dekker, New York.

3. Chopineau, J., McCafferty, F.D., Therisod, M., Klibanov, A. M. 1988. Production of biosurfactants from sugar alcohols and vegetable oil catalyzed by lipases in nonaqueous medium. Biotechnol. Bioeng. 31: 208-214.

4. Fletcher, P.D.I., Freedman, R. B., Robinson, B. H., Rees, G. D., Schomacker, R. 1987. Lipase-catalyzed ester synthesis in oil-continuous microemulsions. Biochim. Biophys. Acta 912: $278-282$.

5. Goderis, H., Ampe, G., Feyten, M., Fouwe, B., Guffens, W., Cauwenbergh, S.V., Tobback, P. 1987. Lipase-catalyzed ester exchange reactions in organic media with controlled humidity. Biotechnol. Bioeng. 30: 258-266.

6. Hajjar, A. B., Nicks, P. F., Knowles, C. J. 1990. Preparation of monomeric acrylic ester intermediates using lipase catalyzed transesterifications in organic solvents. Biotechnol. Lett. 12: 825-830.

7. Hayes, D. G. 1991. Esterification reactions catalyzed by lipases in reverse micellar media. Ph.D. Thesis, University of Michigan.
8. Hayes, D. G., Gulari, E. 1990. Esterification reactions of lipase in reverse micelles. Biotechnol. Bioeng. 35: 793-801.

9. Hayes, D. G., Gulari, E. 1991. To appear.

10. Hayes, D. G., Gulari, E. 1991. 1-monoglyceride production from lipase-catalyzed esterification of glycerol and fatty acid in reverse micelles. Biotechnol. Bioeng. 38: 507-517.

11. Holmberg, K., Österberg, E. 1987. Enzymatic transesterification of a triglyceride in microemulsions. Progr. Coll. Polym. Sci. 74: 98-102.

12. Idson, B. 1985. Surfactants for cosmetic microemulsion. pp. 128 In: M. M. Rieger (ed.), Surfactants in cosmetics: surfactant science series, vol. 6. Marcel Dekker, New York.

13. Kaimal, T., Saroja, M. 1989. The active site composition of porcine pancreatic lipase: possible involvement of lysine. Biotechnol. Biophys. Acta 999: 331-334.

14. Langrand, G., Baratti, J., Buono, G., Triantaphylides, C. 1988. Enzymatic separation and resolution of nucleophiles: a predictive kinetic model. Biocatalysis 1: 231-248.

15. Luisi, P. L., Giomini, M., Pileni, M. P., Robinson, B. H. 1988. Reverse micelles as hosts for proteins and small molecules. Biochim. Biophys. Acta 947: 209-246.

16. Margolin, A. L., Crenne, J.Y., Klibanov, A. M. 1987. Stereoselective oligomerization catalyzed by lipases in organic solvents. Tetrahedr. Lett. 28: 1607-1610.

17. McNeil, G.P., Shimizu, S., Yamane, T. 1991. High yield enzymatic glycerolysis of fats and oils. J. Am. Oil Chem. Soc. 68: 1-5.

18. Neidman, S. L. 1987. Surfactants and biotechnology. pp. 131144 In: M. J. Rosen (ed.), Surfactants in emerging technologies: surfactant science series, vol. 26. Marcel Dekker, New York.

19. Oldfield, C., Rees, G. D., Robinson, B. H., Freedman, R. B. 1987. Enzyme action and enzyme reversal in water-in-oil microemulsions. pp. 119-123 In: C. Laane, J. Tramper, and M.D. Lilly (eds.), Biocatalysis in organic media. Elsevier, Amsterdam.

20. Swern, D. (ed.) 1964. Bailey's industrial oil and fat products. J. Wiley, New York.

21. Zaks, A., Klibanov, A. M. 1984. Enzymatic catalysis in organic media at $100^{\circ} \mathrm{C}$. Science 224: $1249-1251$. 